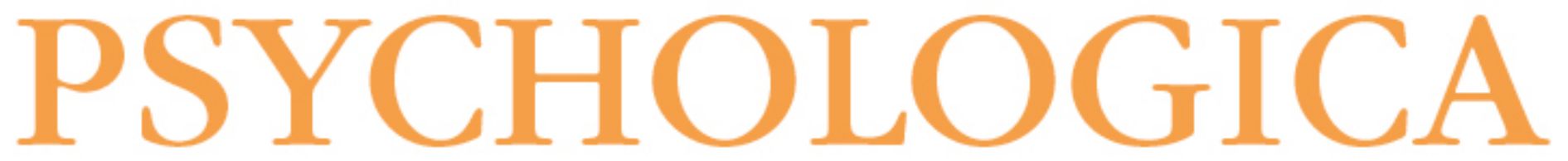

\title{
Perceção do apoio dos professores no desenvolvimento de carreira das crianças e jovens adolescentes: validação de uma escala de avaliação
}

Autor(es): $\quad$ David, Rute; Silva, José Tomás da; Paixão, Maria Paula

Publicado por: Imprensa da Universidade de Coimbra

URL

persistente: URI:http://hdl.handle.net/10316.2/41447

DOI: DOI:https://doi.org/10.14195/1647-8606_59_2_5

Accessed : $\quad$ 26-Apr-2023 10:36:36

A navegação consulta e descarregamento dos títulos inseridos nas Bibliotecas Digitais UC Digitalis, UC Pombalina e UC Impactum, pressupõem a aceitação plena e sem reservas dos Termos e Condições de Uso destas Bibliotecas Digitais, disponíveis em https://digitalis.uc.pt/pt-pt/termos.

Conforme exposto nos referidos Termos e Condições de Uso, o descarregamento de títulos de acesso restrito requer uma licença válida de autorização devendo o utilizador aceder ao(s) documento(s) a partir de um endereço de IP da instituição detentora da supramencionada licença.

Ao utilizador é apenas permitido o descarregamento para uso pessoal, pelo que o emprego do(s) título(s) descarregado(s) para outro fim, designadamente comercial, carece de autorização do respetivo autor ou editor da obra.

Na medida em que todas as obras da UC Digitalis se encontram protegidas pelo Código do Direito de Autor e Direitos Conexos e demais legislação aplicável, toda a cópia, parcial ou total, deste documento, nos casos em que é legalmente admitida, deverá conter ou fazer-se acompanhar por este aviso. 
VOLUME

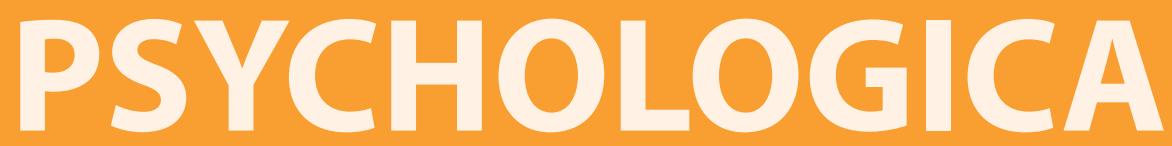

IMPRENSA DA UNIVERSIDADE DE COIMBRA

COIMBRA UNIVERSITY PRESS

FACULDADE DE PSICOLOGIA E DE CIÊNCIAS

DA EDUCAÇÃO DA UNIVERSIDADE DE COIMBRA 


\title{
Perceção do apoio dos professores no desenvolvimento de carreira das crianças e jovens adolescentes: Validação de uma escala de avaliação ${ }^{1}$
}

\author{
Rute David ${ }^{2}$, José Tomás da Silva ${ }^{3}$ e Maria Paula Paixão ${ }^{4}$
}

Perceived teacher support on children and adolescent career development: Validation of a rating scale

\begin{abstract}
Specialized literature has been lately emphasizing the importance of the relational context in the career development process. Being school a meaningful context to children and young adolescents, we focused on the influence of teachers, specifically in the students' perceptions of teachers' support to their career development process, particularly in what concerns to their interests, perceptions of competence and, educational and occupational aspirations. Given the lack of valid measures to assess this construct, this study presents data from the validation of the translation and adaptation of the Portuguese version of the Teacher Support Scale (McWhirter, 1996). In a study with 498 participants from the $4^{\text {th }}$ $6^{\text {th }}$ and $8^{\text {th }}$ grades, we found differences in teacher support perceptions, based on gender, school level (age), socioeconomic status and level of academic aspirations.
\end{abstract}

Keywords: career development; children and young adolescents'; teacher support

1 Trabalho realizado no âmbito da bolsa de Doutoramento (SFRH / BD / 68364 / 2010) financiada pela Fundação para Ciência e a Tecnologia, Portugal.

2 Faculdade de Psicologia e Ciências da Educação da Universidade de Coimbra, Coimbra, Portugal. E-mail: rute.david@fpce.uc.pt

3 Faculdade de Psicologia e Ciências da Educação da Universidade de Coimbra, Coimbra, Portugal

E-mail: jtsilva@fpce.uc.pt

4 Faculdade de Psicologia e Ciências da Educação da Universidade de Coimbra, Coimbra, Portugal

E-mail: mppaixao@fpce.uc.pt

Artigo recebido a 01-07-2016 e aprovado a 06-09-2016. 


\section{Resumo}

A literatura especializada tem vindo a enfatizar a importância do contexto relacional no desenvolvimento de carreira. Sendo a escola um contexto significativo da vida das crianças e jovens adolescentes, debruçámo-nos sobre a influência dos professores, mais especificamente, na perceção de apoio dos professores por parte dos alunos no seu processo de desenvolvimento de carreira, nomeadamente ao nível dos interesses, perceções de competência e aspirações educativas e profissionais. Face à escassez de medidas deste constructo, apresentamos, neste artigo, dados da validação da tradução e adaptação da versão portuguesa da Teacher Support Scale (McWhirter, 1996). Num estudo com 498 participantes do Ensino Básico ( $4^{\circ}, 6^{\circ}$ e $8^{\circ}$ anos de escolaridades) encontrámos diferenças na perceção do apoio dos professores em função do sexo, nível de escolaridade (idade), nível socioeconómico e nível de aspirações académicas.

Palavras-chave: desenvolvimento de carreira; crianças e jovens adolescentes; apoio dos professores

\section{INTRODUÇÃO}

A importância da influência do contexto relacional no desenvolvimento de carreira tem vindo a ser destacada em vários estudos emergentes na área da psicologia vocacional (Blustein, 2011; Schultheiss, 2003), realçando a necessidade de se reconhecer o significado da esfera relacional na vida das pessoas e o apoio de figuras significativas na tomada de decisão de carreira e nas trajetórias de desenvolvimento (Abreu, 1996; Metheny, McWhirter, \& O’Neil, 2008). A conceptualização do trabalho enquanto um ato relacional (Blustein, 2011) implica a consideração do impacto de outras pessoas no processo de desenvolvimento de carreira do sujeito, em detrimento de um processo de formulação de projetos e escolhas profissionais feito de forma relativamente isolada do contexto relacional do sujeito, veiculado por algumas posições teóricas (Blustein, 2011).

Apesar de as variáveis contextuais serem há muito consideradas como fatores importantes na modelação do comportamento vocacional, só mais recentemente se incidiu na experiência relacional enquanto fator de contexto (Metheny et al., 2008). A Teoria Sociocognitiva de Carreira (e.g., Lent, Brown, \& Hacket, 2000) tem vindo a destacar a importância de figuras significativas no ambiente do sujeito (fatores externos) e a sua influência nas expetativas de autoeficácia de carreira e de resultados positivos (fatores internos), bem como na transformação de interesses 
de carreira em objetivos e ações (Kenny \& Medvide, 2013; Lent, Paixão, Silva, \& Leitão, 2010; Metheny et al., 2008). A abordagem desenvolvimentista-contextualista (Vondracek, Lerner, \& Schulenberg, 1986) é outra teoria que igualmente realça a interdependência entre variáveis pessoais e contextuais no desenvolvimento vocacional. Recentemente, outras teorias ou modelos emergentes têm vindo a conceptualizar a vertente relacional como essencial no trabalho e na vida, quer ao nível da construção dos significados atribuídos ao trabalho, quer ao nível do processo dialógico de construção da identidade vocacional (Blustein, 2011; Duffy, Blustein, Diemer, \& Autin, 2016; Savickas, 2005; Schultheiss, 2003).

A literatura no âmbito da psicologia vocacional tem vindo a aprofundar o conhecimento do papel dos aspetos relacionais no desenvolvimento de carreira das crianças e adolescentes (Schultheiss, 2007). As pesquisas nesta temática têm evidenciado o contributo da influência dos pais (e família), dos colegas de escola, dos amigos, dos professores ou dos psicólogos e conselheiros escolares (Abreu, 1996; Kenny \& Bledsoe, 2005; Paa \& McWhirter, 2000; Schultheiss, Palma, \& Manzi, 2005; Wall, Covell, \& MacIntyre, 1999), nas expetativas e valores de carreira, autoeficácia e interesses, e na busca de objetivos educacionais e de carreira (Schultheiss, 2007).

Sendo a escola um dos contextos mais proeminentes no dia a dia das crianças e jovens, iremo-nos debruçar neste trabalho, especificamente, sobre a influência dos professores, porquanto, citando Abreu (1996)

(...) em tudo o que diz respeito ao comportamento dos alunos, desde o desenvolvimento cognitivo ao desenvolvimento vocacional, os professores encontram-se implicados ou envolvidos [...] Fazem parte do mundo dos alunos, interactuam com eles de forma privilegiada, sistemática, quase quotidiana [...] "Integram" a sua personalidade, influenciam, de forma positiva ou negativa, o seu desenvolvimento. (p. 115)

Apesar de a maioria dos estudos incidir sobre o rendimento académico, a centralidade do papel dos professores no desenvolvimento de carreira dos alunos também tem sido destacada pela literatura, tanto a nível nacional como internacional (Ferreira, Nascimento, \& Fontaine, 2009; Gamboa \& Paixão, 2014; Metheny et al., 2008, Oliveira, Taveira, \& Neves, 2014).

As crianças desenvolvem os seus interesses e valores através da interação com os professores (bem como com os membros da família, grupo de pares e media) e estas transações influenciam as suas conceções gerais acerca do trabalho, as suas aspirações e expetativas de carreira, bem como decisões significativas que tomarão nas suas vidas (Hartung, Porfeli, \& Vondracek, 2005). Os professores são agentes influentes e promotores do desenvolvimento vocacional dos alunos (Ferreira et al., 
2009; Gamboa \& Paixão, 2014), contribuindo para a construção e/ou desconstrução dos seus interesses, para o processo de atribuição de significados acerca do trabalho, nomeadamente quando conferem instrumentalidade aos conteúdos curriculares que lecionam (Oliveira et al., 2014). As crianças e jovens são capazes de distinguir várias fontes de apoio social (e.g., família, grupo de pares, escola) e, quando se sentem apoiadas através de pelo menos uma dessas fontes, revelam-se otimistas em relação às suas vidas e acreditam terem mais controlo sobre as mesmas, tanto no presente como no futuro (Dubow \& Ullman, 1989; Ryan, Stiller, \& Lynch, 1994). Os adolescentes com níveis elevados de apoio social percebem mais facilmente as oportunidades, acreditam que terão acesso a ocasiões de progresso tanto a nível educacional como profissional e antecipam o sucesso futuro (Wall et al., 1999). O apoio dos professores, tal como o dos pares, complementa o apoio parental, e pode ser compensatório na ausência de um ambiente familiar em que o aluno não se sinta apoiado (Perry, Liu, \& Pabian, 2010; Wall et al., 1999), constituindo-se como um fator protetor que promove a preparação de carreira, reduzindo o risco de desinvestimento escolar (Perry et al., 2010).

Os resultados de um estudo com crianças dos $4^{\circ}$ e $5^{\circ}$ anos de escolaridade, desenvolvido por Schultheiss et al. (2005), indicam que os professores são vistos pelos alunos como: influentes na aquisição de conhecimento e informação acerca das profissões, aparecendo como agentes educativos que fornecem apoio social, que identificam pontos fortes nos alunos, nomeadamente capacidades e a respetiva ligação ao desempenho de potenciais profissões futuras, sendo vistos como figuras-modelo, que criam experiências de exploração, que moldam as conceções de trabalho dos alunos e que enfatizam a importância da escola, transmitindo valores de vida. Alunos e professores reconhecem o papel que estes últimos desempenham no desenvolvimento dos alunos em termos pessoais, científicos e pedagógicos (influência geral), a sua ação específica no âmbito da disciplina que lecionam e respetiva ligação a projetos vocacionais, bem como a importância da cooperação dos professores com outros agentes educativos e comunitários (Pinto, Taveira, \& Fernandes, 2003). As pesquisas de Noack, Kracke, Gniewosz e Dietrich (2010) indicam que quanto mais os alunos consideram o clima escolar como sendo de aceitação e de abertura, mais intenso é o comportamento de exploração ocupacional. Uma investigação de Kenny e Bledsoe (2005) evidenciou o papel dos professores na identificação dos jovens com a escola, sugerindo que estes podem contribuir positivamente para a forma como os primeiros valorizam o contexto escolar, para além do impacto exercido pelas crenças da família, dos amigos próximos e do grupo de pares. Dados de um estudo de Metheny et al. (2008) indicam que a perceção do apoio dos professores (e.g., ajuda no sucesso académico, transmissão de expetativas elevadas de sucesso escolar e acessibilidade como formas de apoio e de informação de carreira) está positivamente associada à autoeficácia na tomada de decisão de 
carreira e às expetativas de resultados vocacionais positivos (Kenny \& Medvide, 2013). O estudo realizado por Perry et al. (2010) evidenciou a contribuição significativa do papel dos professores na preparação de carreira de alunos de meios urbanos desfavorecidos, para além da influência positiva igualmente identificada em termos de envolvimento escolar.

No entanto, as interações entre professores e alunos nem sempre são positivas e, por vezes, podem mesmo ter repercussões negativas no desenvolvimento académico e social dos jovens, logo nos primeiros anos de escolaridade (Kenny \& Medvide, 2013). Um estudo de Mercer e DeRosier (2008) evidenciou que, ao nível do $3^{\circ}$ ano de escolaridade, baixas preferências individuais dos professores em relação aos alunos estariam associadas à rejeição pelos pares, baixo desempenho académico e sintomas de depressão durante o $1^{\circ}$ ciclo. Ao nível do ensino secundário, um estudo de Bae, Holloway, Li e Bempechat (2008) sugere que os alunos, especialmente aqueles com baixo rendimento académico, começam a distanciar-se da escola quando percecionam que as expetativas dos professores, relativamente ao seu comportamento na aula e desempenho, são baixas.

As pesquisas, para além do que já foi referido, têm igualmente demonstrado o efeito de variáveis como o sexo e a idade na perceção de apoio dos professores por parte dos alunos. As raparigas tendem a evidenciar perceções de apoio mais elevadas do que os rapazes (Dubow \& Ullman, 1989; Malecki \& Elliot, 1999; Ryan et al., 1994; Wall et al., 1999) e, nestas, estas perceções parecem estar relacionadas com a antecipação de oportunidades de educação e de carreira, o que não se verificou nos rapazes (Wall et al., 1999). Outro dado interessante é que à medida que aumenta a idade dos alunos parecem diminuir as perceções de apoio em relação aos professores (Malecki \& Elliot, 1999).

Apesar de o reconhecimento da importância do contexto relacional em termos concetuais e de intervenção se estar a expandir na literatura (Blustein, 2011), são necessários mais estudos neste âmbito que possam elucidar de que forma os professores (e a família) têm influência no desenvolvimento de carreira dos alunos (Kenny \& Medvide, 2013; Pinto et al., 2003). A falta de instrumentos de avaliação fundamentais a estas pesquisas é uma razão que, frequentemente, é apontada para justificar a incipiência da investigação neste domínio. O presente trabalho procura colmatar essa lacuna ao nível da instrumentação ao examinar as características psicométricas de uma medida das perceções que os alunos têm do apoio aos seus projetos de carreira, por parte dos seus professores.

O estudo que efetuámos faz parte de uma investigação longitudinal mais abrangente sobre as aspirações vocacionais na infância. Os dados apresentados foram recolhidos no terceiro e último momento de avaliação, do estudo longitudinal, e são referentes a um corte transversal da amostra. Iremos abordar a influência de variáveis como 
o sexo, a idade e o nível socioeconómico de pertença dos alunos, ao apresentarmos a tradução e adaptação da versão portuguesa da Teacher Support Scale (TSS).

\section{MÉTODO}

\section{Participantes}

A amostra deste estudo é composta por 498 participantes, a frequentar os $4^{\circ}, 6^{\circ}$ e $8^{\circ}$ anos de escolaridade em estabelecimentos de ensino público, ensino particular e cooperativo da Região Centro de Portugal Continental, sendo 250 alunos do sexo masculino (50.2\%) e 248 (49.8\%) do sexo feminino. Relativamente ao nível socioeconómico (NSE), de acordo com a classificação delineada por Simões (1994), 32.7\% dos alunos são provenientes de NSE Baixo, 38.5\% de NSE Médio e 29.1\% de NSE Elevado.

\section{Instrumento}

A Teacher Support Scale (McWhirter, 1996) é uma escala composta por 27 itens, construída para avaliar as perceções dos alunos acerca do apoio dos seus professores do ensino secundário. Os itens da versão original foram desenvolvidos com base em revisão bibliográfica existente na área e aprovados por um painel de professores. Os itens são avaliados através de uma escala com cinco alternativas de resposta que vão desde $\mathrm{A}=$ concordo fortemente a $\mathrm{E}$ = discordo. Apesar de a TSS se destinar originalmente a avaliar as perceções dos alunos relativamente ao apoio percebido por parte dos professores do ensino secundário, contactámos a autora da versão original que foi da opinião de que poderia ser utilizada com alunos mais novos, tendo, para isso, de ser necessária a adaptação da formulação original das questões. Dada a relevância da utilização desta escala no nosso estudo, bem como a escassez de instrumentos deste tipo desenvolvidos especificamente para alunos mais novos, considerámos que a aplicação da TSS seria um contributo muito importante na nossa pesquisa. Após obtermos a permissão da autora para a tradução e a utilização da escala original norte-americana no nosso estudo, procedemos à tradução e adaptação para a língua portuguesa. Neste processo contámos com a colaboração de uma psicóloga com vários anos de experiência em contexto escolar. Fizemos algumas adaptações para facilitar a compreensão de determinados itens por parte 
das crianças mais novas, por conterem vocabulário com o qual não estavam muito familiarizadas. A tradução foi revista por investigadores experientes e submetida a um grupo de alunos dos $4^{\circ}, 5^{\circ}, 6^{\circ}$ e $8^{\circ}$ anos de escolaridade. Não detetámos dificuldades na compreensão das instruções e dos itens que traduzimos e adaptámos.

Os resultados de um estudo de Metheny et al. (2008) em que participaram 325 alunos finalistas do ensino secundário e no qual foi utilizada uma versão reduzida da TSS com 21 itens, indicam a existência de quatro fatores: 1) investimento, 2) consideração positiva, 3) expetativas, 4) acessibilidade. $O$ primeiro fator (oito itens), designado Investimento, diz respeito à perceção de que os professores se envolvem na facilitação (ajuda) de comportamentos direcionados para o sucesso futuro e desempenho dos alunos. O segundo fator (cinco itens), Consideração positiva, está relacionado com a perceção de que os professores se preocupam e estão ligados emocionalmente e disponíveis para os alunos. O terceiro fator (cinco itens), Expetativas, contempla a perceção de que os professores transmitem expetativas positivas em relação ao sucesso educativo dos alunos. Finalmente o quarto fator (três itens), Acessibilidade, refere-se à perceção relativamente à disponibilidade do professor e à sua recetividade à busca de apoio ou informação por parte dos alunos.

Apresentamos de seguida os resultados da tradução e adaptação da versão portuguesa da TSS.

\section{Análise de dados}

Para uma primeira estimativa da distribuição dos itens pelos fatores e a magnitude da sua associação, recorremos à Análise Fatorial Exploratória (AFE) da matriz das correlações, com extração dos fatores pelo método da fatorização do eixo principal com rotação oblíqua Promax. Inicialmente foram extraídos quatro fatores, mas só foram retidos para análise posterior dois fatores comuns que apresentavam um valor próprio superior a 1 [resultado que é consonante com o teste da escarpa sedimentar (scree plot), e que foi ainda corroborado pela análise do método da análise paralela (Paralell Analysis: PA)]. Para determinar a validade da realização da AFE utilizaram-se os critérios habitualmente referidos na literatura. Na medida KMO [Kaiser-Meyer-Olkin], observou-se um valor de .95, considerado excelente de acordo com os critérios de classificação definidos em Marôco (2014a). O teste de esfericidade de Bartlett apresenta um $p$-value $<.001$, demonstrativo de que as variáveis estão significativamente correlacionadas e que é apropriado executar a AFE nos dados. Na totalidade estes dois fatores explicaram $50.3 \%$ de variância comum.

Seguidamente fez-se uma nova análise forçada a dois fatores e depois de eliminados os loadings abaixo de .40. Assim, o fator 1 apresenta loadings salientes em 14 
itens e o fator 2 em oito itens. No entanto, a distribuição dos itens pelos fatores não permitiu distinguir dimensões semelhantes às que foram encontradas em estudos com a versão original da escala. Detetámos que no segundo fator se agrupavam os três itens do fator acessibilidade, mas em conjunto com itens relacionados com o fator de investimento e de consideração positiva.

De seguida, procedemos a uma análise fatorial confirmatória (AFC) com os 22 itens que se revelaram mais promissores na AFE, utilizando o software AMOS (v. 22, SPSS, da IBM Company Chicago, IL). O modelo bi-fatorial da adaptação portuguesa da TSS para uma amostra de 498 alunos, com 22 itens, revelou uma qualidade de ajustamento sofrível, de acordo com os critérios de classificação definidos em Marôco (2014b) [ $\chi^{2}$ / $g l=4.372 ; C F I=.887 ; G F I=.859 ;$ RMSEA $=.082 ;$ p $($ rmsea $\leq .05)<.001]$. Depois de eliminados os itens com loadings inferiores a .60 obtidos na EFA (itens 5, 9, 11, 12, 15 , 19, 10, 14, 20, e 26), e correlacionados os erros de medida dos itens 6, 7, 18, 21, 23, 24 e 27, foi possível obter uma qualidade de ajustamento aceitável $[\chi 2 / g l=2.606$; $C F I=$ $.973 ; G F I=.960 ; R M S E A=.057 ; p[$ rmsea $\leq .05)=.168]$ com os 12 itens remanescentes: oito itens no fator 1 (que designámos Apoio Académico) e quatro itens no fator 2 (que designámos Apoio Social). Cada variável observada (item) está associada unicamente a uma das duas variáveis latentes mencionadas. Apresentamos o modelo final na Figura 1 , onde se podem observar os valores dos coeficientes de regressão estandardizados e a fiabilidade individual de cada um dos itens no modelo final simplificado.

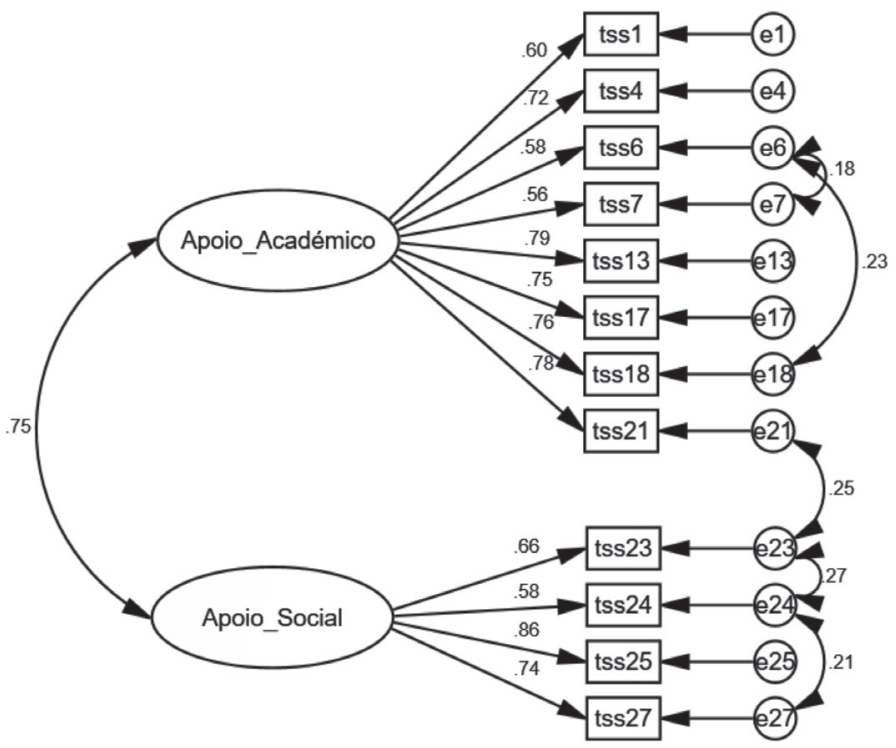

Figura 1. Modelo Bi-fatorial Ajustado à Amostra de 498 Alunos do Ensino Básico $[\chi 2 / \mathrm{gl}=2.61$; $\mathrm{CFI}=.973 ; \mathrm{GFI}=.960 ; \mathrm{RMSEA}=.057 ; \mathrm{p}(\mathrm{rmsea}<.05)=.168]$. 
O fator Apoio Académico engloba itens mais relacionados com as aprendizagens escolares, tais como: "esperam que eu seja aplicado/a (trabalhe muito) na escola", "disponibilizam-se para me ajudar a ter melhores notas", "incentivam-me a aprender", enquanto o fator Apoio Social ${ }^{5}$ integra itens de índole mais relacional, que não estão diretamente associados ao contexto académico, como: "falam comigo sobre o que eu desejo para o meu futuro (sobre as minhas aspirações futuras)", "estão disponíveis para falar comigo sobre outros assuntos para além da escola”.

O grau de precisão das respostas nos itens de cada fator foi calculado com base no coeficiente alfa de Cronbach. Também estimámos este índice para o conjunto dos itens. O cômputo da pontuação global justifica-se pelo valor elevado da correlação interfatores $(r=.75)$. Com efeito, considerando o total da escala (12 itens) a consistência interna é de $.90^{6}$, no Fator 1 (oito itens) é de .88 e no Fator 2 (quatro itens) de .83. Os resultados sugerem uma boa consistência interna do instrumento e são consonantes com os encontrados noutros estudos em que foi utilizada a versão original da TSS. Com efeito, considerando a escala total, Metheny et al. (2008) obtiveram um alfa de .96, enquanto no estudo Perry et al. (2010) esse valor foi de .94.

Considerando o total da amostra, as pontuações médias obtidas pelos 498 participantes situam-se, tanto na subescala Apoio Académico $(M=4.22, D P=0.64)$ como na subescala Apoio Social $(M=3.71, D P=0.81)$, acima do ponto médio (3) da escala de cinco pontos, acontecendo o mesmo em todos os 12 itens da escala (Tabela 1).

Tabela 1

Médias e Desvios Padrão dos 12 Itens Remanescentes da Versão Portuguesa da TSS

\begin{tabular}{llccc}
\hline Item & Fator & $M$ & $D P$ & Correlação item-total \\
\hline Item 1 & Apoio Académico & 4.28 & 0.81 & .57 \\
Item 4 & Apoio Académico & 4.26 & 0.90 & .67 \\
Item 6 & Apoio Académico & 4.40 & 0.74 & .59 \\
Item 7 & Apoio Académico & 3.83 & 0.94 & .55 \\
Item 13 & Apoio Académico & 4.24 & 0.88 & .75 \\
Item 17 & Apoio Académico & 4.19 & 0.88 & .69 \\
Item 18 & Apoio Académico & 4.39 & 0.78 & .72 \\
Item 21 & Apoio Académico & 4.14 & 0.90 & .71 \\
Item 23 & Apoio Social & 3.86 & 0.90 & .63 \\
Item 24 & Apoio Social & 3.44 & 1.04 & .64 \\
Item 25 & Apoio Social & 3.88 & 0.98 & .69 \\
Item 27 & Apoio Social & 3.66 & 1.05 & .69 \\
\hline
\end{tabular}

5 Esta designação baseia-se na conceptualização de "apoio social" avançada por Cutrona (1996) que integra vários aspetos multidimensionais do mesmo (i.e. emocional, integração social, estima, tangibilidade e informação) e que foi utilizada no estudo de Schultheiss et al. (2005) acima referido.

$6 \mathrm{Na}$ interpretação deste coeficiente deve ter-se em conta que o número de itens da versão Portuguesa é menor. 
Quando desagregada a amostra em função do sexo dos participantes, verificamos a existência de diferenças estatisticamente significativas entre rapazes e raparigas (Tabela 2 ).

Tabela 2

Comparação das Diferenças das Médias por Sexo

\begin{tabular}{lcccccc}
\hline & \multicolumn{2}{c}{$\begin{array}{c}\text { Rapazes } \\
(n=250)\end{array}$} & \multicolumn{2}{c}{$\begin{array}{c}\text { Raparigas } \\
(n=248)\end{array}$} & & \\
& $M$ & $D P$ & $M$ & $D P$ & & \\
\cline { 2 - 5 } & 4.13 & 0.69 & 4.30 & 0.57 & -3.04 & .002 \\
Apoio Académico & 3.73 & 0.89 & 3.70 & 0.72 & 0.38 & NS \\
Apoio Social & 4.00 & 0.69 & 4.10 & 0.53 & -1.90 & NS \\
Escala Total & & & & & & \\
\hline
\end{tabular}

Relativamente à subescala Apoio Académico as raparigas obtêm pontuações médias mais elevadas do que os rapazes, sendo essa diferença estatisticamente significativa, e a magnitude do efeito média $[t(496)=-3.04, p=.002, d=0.27]$ (Marôco, 2014a). Já na subescala Apoio Social, os rapazes demonstram perceções de apoio por parte dos professores mais elevadas do que as raparigas, mas essa diferença não assume significância estatística $[t(496)=0.38, p=.71, d=0.03]$. Considerando a escala total, não existem diferenças significativas entre rapazes e raparigas $[t(496)=-1.90, p=.06, d=0.17]$.

Considerando os três níveis de ensino da amostra ( $4^{\circ}, 6^{\circ}$ e $8^{\circ}$ anos de escolaridade), verificamos que as pontuações médias tendem a baixar com a progressão na escolaridade, tendência já evidenciada noutros estudos (Malecki \& Elliot, 1999) (Tabela 3).

Tabela 3

Comparação das Diferenças das Médias por Ano de Escolaridade

\begin{tabular}{lccccccccc}
\hline & $\begin{array}{c}4 \text { ano } \\
(n=162)\end{array}$ & $\begin{array}{c}6^{\circ} \text { ano } \\
(n=196)\end{array}$ & $\begin{array}{c}8^{\circ} \text { ano } \\
(n=140)\end{array}$ & $F$ & $p$ & $\begin{array}{c}\text { Teste } \\
\text { post-hoc }\end{array}$ \\
\cline { 2 - 6 } & $M$ & $D P$ & $M$ & $D P$ & $M$ & $D P$ & & & \\
\hline A. Académico & 4.38 & 0.64 & 4.19 & 0.58 & 4.07 & 0.68 & 9.76 & $<.001$ & $4>6 ; 4>8$ \\
A. Social & 3.89 & 0.82 & 3.67 & 0.77 & 3.56 & 0.83 & 6.81 & $<.001$ & $4>6 ; 4>8$ \\
Escala Total & 4.22 & 0.62 & 4.02 & 0.57 & 3.90 & 0.67 & 10.65 & $<.001$ & $4>6 ; 4>8$ \\
\hline
\end{tabular}


As análises de variância (ANOVA) revelaram a existência de diferenças estatisticamente significativas entre as médias dos três níveis de ensino: Apoio Académico: $F(2,495)=9.76, p<.001, \eta_{p}^{2}=.04 ;$ Apoio Social: $F(2,495)=6.81, p=.001, \eta_{p}^{2}=$ .03 ; Escala Total: $F(2,495)=10.65, p<.001, \eta_{p}^{2}=.04$. Examinando os resultados dos testes post-hoc (teste de Bonferroni), verificamos a existência de diferenças significativas quer ao nível da escala total, quer ao nível das duas subescalas entre os alunos mais novos ( $4^{\circ}$ ano) e os alunos a frequentar os $6^{\circ}$ e $8^{\circ}$ anos de escolaridade, sendo a magnitude do efeito pequena. Apesar de os alunos do $6^{\circ}$ ano de escolaridade exibirem pontuações médias mais elevadas do que os alunos do $8^{\circ}$ ano, essas diferenças não assumem significância em termos estatísticos.

Considerando o nível socioeconómico (NSE) dos participantes do nosso estudo, encontrámos diferenças estatisticamente significativas na subescala Apoio Académico e na Escala Total (Tabela 4).

Tabela 4

Comparação das Diferenças das Médias por Nível Socioeconómico (NSE)

\begin{tabular}{|c|c|c|c|c|c|c|c|c|c|}
\hline & \multicolumn{2}{|c|}{$\begin{array}{c}\text { NSE B } \\
(n=163)\end{array}$} & \multicolumn{2}{|c|}{$\begin{array}{c}\text { NSE M } \\
(n=190)\end{array}$} & \multicolumn{2}{|c|}{$\begin{array}{c}\text { NSE E } \\
(n=145)\end{array}$} & \multirow{2}{*}{$F$} & \multirow{2}{*}{$p$} & \multirow{2}{*}{$\begin{array}{c}\text { Teste } \\
\text { post-hoc }\end{array}$} \\
\hline & $M$ & $D P$ & $M$ & $D P$ & $M$ & $D P$ & & & \\
\hline A. Académico & 4.03 & 0.59 & 4.21 & 0.70 & 4.42 & 0.54 & 15.13 & $<.001$ & $\mathrm{~B}<\mathrm{M}<\mathrm{E}$ \\
\hline A. Social & 3.69 & 0.78 & 3.69 & 0.86 & 3.76 & 0.78 & 0.34 & NS & - \\
\hline Escala Total & 3.92 & 0.59 & 4.04 & 0.69 & 4.20 & 0.53 & 8.01 & $<.001$ & $\begin{array}{l}\mathrm{B}<\mathrm{E} \\
\mathrm{M}<\mathrm{E}\end{array}$ \\
\hline
\end{tabular}

De um modo geral, as perceções de apoio dos professores tendem a ser mais elevadas consoante o nível socioeconómico (NSE) a que pertencem os alunos. Os participantes provenientes de NSE elevado obtêm pontuações médias mais elevadas, considerando a escala total e as subescalas Apoio Académico e Apoio Social, do que os restantes participantes no estudo. Na subescala Apoio Académico foram registadas diferenças estatisticamente significativas entre as pontuações médias dos participantes provenientes dos três níveis socioeconómicos (baixo, médio e elevado), aumentando as pontuações à medida que aumenta o NSE, sendo a dimensão do efeito média [Apoio Académico: $F(2,495)=15.13, p<.001, \eta_{p}{ }^{2}=.06$ ]. Considerando a escala total, foram encontradas diferenças estatisticamente significativas entre os alunos de NSE elevado e NSE baixo e entre os alunos de NSE elevado e NSE médio, com baixa magnitude de efeito [Escala Total: $F(2,495)=8.01, p<.001, \eta_{p}{ }^{2}=.03$ ] Apesar de os participantes provenientes de NSE médio apresentaram perceções 
de apoio relativamente aos professores da sua escola mais elevadas do que os participantes de NSE baixo, essas diferenças não são estatisticamente significativas. As perceções de apoio relativas à subescala Apoio Social não foram influenciadas pelo nível de NSE de pertença dos alunos, como sugere a interação não significativa entre os dois fatores [Apoio Social: $F(2,495)=0.34, p=.71, \eta_{p}{ }^{2}=.001$ ].

Ao questionarmos os participantes do nosso estudo acerca do nível de ensino que pretendem completar, definimos dois níveis de aspirações educacionais: elevadas (quando pretendem completar estudos a nível superior) e baixas (quando pretendem apenas concluir a escolaridade obrigatória); 21 participantes não sabem ou não responderam a esta questão e foram excluídos da análise. Encontrámos diferenças ao desagregar a amostra em função das aspirações educacionais dos alunos (Tabela 5).

Tabela 5

Comparação das Diferenças das Médias por Aspirações Educacionais

\begin{tabular}{lcccccc}
\hline & $\begin{array}{c}\text { Ensino Secundário } \\
(n=108)\end{array}$ & \multicolumn{2}{c}{$\begin{array}{c}\text { Estudos Superiores } \\
(n=369)\end{array}$} & $t$ & $p$ \\
\cline { 2 - 4 } & $M$ & $D P$ & $M$ & $D P$ & & \\
\hline Apoio Académico & 4.00 & 0.55 & 4.31 & 0.61 & -4.76 & $<.001$ \\
Apoio Social & 3.64 & 0.74 & 3.74 & 0.82 & -1.19 & NS \\
Escala Total & 3.88 & 0.55 & 4.12 & 0.60 & -3.72 & $<.001$ \\
\hline
\end{tabular}

São os alunos que pretendem completar estudos a nível superior os que obtêm pontuações médias mais elevadas em todas a escalas, sendo essas diferenças significativas na subescala Apoio Académico $[t(475)=-4.76, p<.001, d=0.52]$ com dimensão do efeito elevada; e na escala total $[t(475)=-3.72, p<.001 ; d=0.41]$ com magnitude de efeito média (Marôco, 2014a). Relativamente à subescala Apoio Social não foram encontradas diferenças estatisticamente significativas entre os dois níveis de ensino que os alunos aspiram completar.

\section{DISCUSSÃO DOS RESULTADOS}

Em termos das propriedades da medida examinada neste estudo validámos um constructo geral que traduz a perceção do aluno acerca do apoio recebido por parte dos professores, mas neste momento não há evidência que apoie os 4 fatores obtidos 
em estudos anteriores com a versão original da TSS. De notar que Metheny et al. (2008) reportaram a existência de um fator de primeira ordem na TSS, sugerindo a existência de um constructo comum a todas as dimensões.

As pontuações obtidas, acima do ponto médio em todos os itens, sugerem que, de um modo geral, os alunos percecionam positivamente o apoio que recebem por parte dos seus professores, com maior incidência no âmbito académico. Estes dados parecem consonantes que as conclusões dos resultados de um estudo de Kenny e Bledsoe (2005) que destaca a contribuição específica do apoio dos professores nas atitudes dos alunos em relação à escola (identificação com a escola), não tendo sido demonstrada nas outras variáveis de adaptabilidade de carreira (perceção de barreiras educacionais, expetativas de resultados de carreira, planeamento de carreira), quando outros fatores foram considerados em primeiro lugar.

Tal como já evidenciado noutros estudos com recurso a outros instrumentos de medida do apoio percebido (Malecki \& Elliot, 1999; Ryan et al., 1994; Wall et al., 1999) as raparigas obtiveram pontuações mais elevadas (considerando a escala total e a subescala Apoio Académico, mas apenas nesta última essas alcançaram o nível de significância estatística) do que os rapazes. Wall e colaboradores (1999) mostraram que as raparigas reportam consistentemente níveis mais elevados de apoio (de fontes para além da família), perceção mais elevada de futuras oportunidades e expetativas mais elevadas tanto a nível de educação como de carreira, bem como aspirações educacionais mais elevadas do que os rapazes; ademais sugeriram que estas diferenças poderão estar relacionadas com o rendimento escolar (geralmente é superior no sexo feminino). Tal assunção parece-nos ir ao encontro dos resultados que obtivemos, já que na subescala Apoio Social não houve diferenças entre rapazes e raparigas (sendo, inclusive, os rapazes a obterem pontuações médias ligeiramente mais elevadas do que as raparigas).

Registámos que com o aumento do nível de escolaridade se verificou uma diminuição nas pontuações médias, tendência que pode estar relacionada com a importância que assumem outros elementos da rede social dos estudantes na formulação das perceções de apoio, nomeadamente o grupo de pares nos alunos mais velhos. Mais uma vez estes resultados estão de acordo com a literatura e com as evidências obtidas noutras pesquisas. Um estudo de Malecki e Elliot (1999), com alunos dos $7^{\circ}$ ao $12^{\circ}$ anos de escolaridade, revelou que a perceção de apoio por parte dos professores diminui com a idade (e nível de ensino), paralelamente ao que acontece relativamente à perceção do apoio dos adultos em geral, sugerindo a existência de diferenças desenvolvimentais nesta variável.

Verificámos que os alunos com aspirações educacionais mais elevadas (que pretendem concluir o ensino superior) têm perceções mais elevadas do apoio dos professores do que os alunos que pretendem apenas concluir estudos a nível 
secundário, sendo essas diferenças significativas, considerando o total da escala e a subescala Apoio Académico. A mesma tendência de resultados parece emergir quando desagregamos a amostra por NSE, evidenciando que são os alunos oriundos de NSE mais elevados que percecionam maiores níveis de apoio por parte dos professores. Devemos sublinhar que grande parte dos alunos com aspirações educacionais mais baixas pertencem, igualmente, a NSE baixo (65.7\%; $28.7 \%$ NSE Médio e 5.6\% de NSE Elevado). Efetivamente, a literatura tem vindo a evidenciar o feito preditor do NSE nas aspirações dos alunos. Os jovens de NSE mais baixo lidam com conflitos entre as normas da escola, família, cultura e os grupos de pares, têm constrangimentos adicionais na formulação de aspirações educativas de nível superior, que incluem limitações físicas (como pressão financeira e distância geográfica) e barreiras psicológicas (Berzin, 2010); deste modo, quando percecionam níveis elevados de barreiras e níveis baixos de apoio demonstram aspirações baixas (Kenny, Blustein, Chaves, Grossman, \& Gallagher, 2003). A pesquisa de Berzin (2010) evidenciou que os alunos de NSE mais baixo reportam, igualmente, níveis baixos de apoio social relativos a várias fontes, incluindo os professores. Esta autora salienta, ainda, que as atribuições positivas ao contexto escolar estão relacionadas com aspirações educacionais elevadas, sugerindo a importância de experiências escolares positivas e de sucesso precoce na amplificação das aspirações.

Nesta equação, é plausível a existência de uma relação estreita entre a perceção de apoio e o sucesso académico (Wall et al., 1999), variável que não estudámos e que tem sido destacada noutras pesquisas (Berzin, 2010; Perry et al., 2010).

\section{CONCLUSÕES}

Dada a relevância do contexto relacional no desenvolvimento de carreira das crianças e jovens, procurámos apresentar dados que contribuam para a compreensão da influência do apoio dos professores neste âmbito.

Concluímos que a perceção deste apoio varia em função do sexo, nível de escolaridade (idade), nível socioeconómico e nível de aspirações académicas, parecendo estar mais relacionado com as aprendizagens escolares (subescala Apoio Académico) e não tanto com o apoio dos professores noutros contextos (subescala Apoio Social). Estando os professores fortemente associados ao contexto de sala de aula e à transmissão de conteúdos no âmbito de disciplinas específicas, é verossímil que a perceção do apoio dos professores por parte dos alunos incida sobretudo nesta vertente.

Em alunos mais novos, a escala parece ter uma estrutura mais reduzida, bem como uma natureza tendencialmente unidimensional (fator de segunda ordem), o 
que poderá estar relacionado com aspetos desenvolvimentais (menor capacidade para diferenciar as perceções de apoio) ou mesmo com diferenças culturais. Um objetivo futuro seria o de aplicar a escala a participantes mais velhos, no sentido de verificar se a estrutura dimensional tende, efetivamente, para uma maior diferenciação.

Finalmente, embora realcemos a necessidade de futuramente clarificar as relações entre as variáveis estudadas, com outras igualmente importantes para a compreensão do processo de desenvolvimento vocacional na infância e na adolescência, nomeadamente com o rendimento escolar, concluímos que a versão portuguesa da TSS pode ser usada, com as adaptações sugeridas, na investigação do comportamento e do desenvolvimento vocacional de crianças e jovens, colmatando desta forma uma lacuna frequentemente referida na literatura especializada.

\section{AGRADECIMENTOS:}

Agradecemos à Susana Igreja Pereira pela colaboração prestada no processo de tradução e adaptação da versão portuguesa da TSS.

\section{REFERÊNCIAS}

Abreu, M. V. (1996). Pais, professores e psicólogos: Contributos para o desenvolvimento de uma prática relacional nas escolas. Coimbra: Coimbra Editora.

Bae, S., Holloway, S. D., Li, J., \& Bempechat (2008). Mexican-American students' perceptions of teachers' expectations: Do perceptions differ depending on student achievement levels? Urban Review, 40(2), 210-225. doi:10.1007/s11256-007-0070-x

Berzin, S. (2010). Educational aspirations among low-income youths: Examining multiple conceptual models. Children \& Schools, 32(2), 112-123. doi:10.1093/cs/32.2.112

Blustein, D. L. (2011). A relational theory of working. Journal of Vocational Behavior, 79(1), 1-17. doi:10.1016/j.jvb.2010.10.004

Cutrona, C. E. (1996). Social support in couples: Marriage as a resource in times of stress. Thousand Oaks, CA: Sage.

Dubow, E. F., \& Ullman, D. G. (1989). Assessing social support in elementary school children: The survey of children's social support. Journal of Clinical Child Psychology, 18(1), 52-64. doi:10.1207/ s15374424jccp1801_7

Duffy, R. D., Blustein, D. L., Diemer, M. A., \& Autin, K. L. (2016). The psychology of working theory. Journal of Counseling Psychology, 63(2), 127-148. doi: 10.1037/cou0000140

Ferreira, A. F., Nascimento, I., \& Fontaine, A. M. (2009). O papel do professor na transmissão de representações acerca de questões vocacionais. Revista Brasileira de Orientação Profissional, 10(2), 43-56. 
Gamboa, V. M., \& Paixão, M. P. (2014). A qualidade da experiência de estágio e o desenvolvimento vocacional de estudantes dos cursos tecnológicos. Psicologia Reflexão e Crítica, 27(2), 377-387. doi:10.1590/1678-7153.201427219

Hartung, P. J., Porfeli, E. J., \& Vondracek, F. W. (2005). Child vocational development: A review and reconsideration. Journal of Vocational Behavior, 66(3), 385-419. doi:10.1016/j.jvb.2004.05.006

Kenny, M. E., \& Bledsoe, M. (2005). Contributions of the relational context to career adaptability among urban adolescents. Journal of Vocational Behavior, 66(2), 257-272. doi: 10.1016/j.jvb.2004.10.002

Kenny, M. E., Blustein, D. L., Chaves, A., Grossman, J. M., \& Gallagher, L. A. (2003). The role of perceived barriers and relational support in the educational and vocational lives of urban high school students. Journal of Counseling Psychology, 50(2), 142-155. doi:10.1037/0022-0167.50.2.142

Kenny, M. E., \& Medvide, M. B. (2013). Relational influences on career development. In S. D. Brown \& R. W. Lent (Eds.), Career development and counseling: Putting theory and research to work ( $2^{\text {nd }}$ ed., pp. 329-356). Hoboken, NJ: John Wiley.

Lent, R. W., Brown, S. D., \& Hackett, G. (2000). Contextual support and barriers to career choice: A social cognitive analysis. Journal of Counseling Psychology, 47(1), 36-49. doi: 10.1037//0022-0167.47.1.36

Lent, R. W., Paixão, M. P., Silva, J. T., \& Leitão, L. M. (2010). Predicting occupational interests and choice aspirations in Portuguese high school students: A test of social cognitive career theory. Journal of Vocational Behavior, 76(2), 244-251. doi: 10.1016/j.jvb.2009.10.001

Malecki, C. K., \& Elliot, S. N. (1999). Adolescents' ratings of perceived social support and its importance: Validation of the Student Social Support Scale. Psychology in the Schools, 36(6), 473-483. doi:10.1002/(SICI)1520-6807(199911)36:6<473::AID-PITS3>3.0.CO;2-0

Marôco, J. P. (2014a). Análise estatística com o SPSS Statistics (6a ed.). Pêro Pinheiro: ReportNumber.

Marôco, J. (2014b). Análise de equações estruturais: Fundamentos teóricos, software \& aplicações (2a ed.). Pêro Pinheiro: ReportNumber.

McWhirter, E. H. (1996). Teacher Support Scale (TSS): A measure of support experienced by high school students from their high school teachers. Unpublished manuscript.

Mercer, S. H. \& DeRosier, M. E. (2008). Teacher preference, peer rejection, and student aggression: A prospective study of transactional influence and independent contributions to emotional adjustment and grades. Journal of School Psychology, 46(6), 661-685. doi:10.1016/j.jsp.2008.06.006

Metheny, J., McWhirter, E. H., \& O'Neil, M. E. (2008). Measuring perceived teacher support and its influence on adolescent career development. Journal of Career Assessment, 16(2), 218 237. doi:10.1177/1069072707313198

Noack, P., Kracke, B., Gniewosz, B., \& Dietrich (2010). Parental and school effects on student's occupational exploration: A longitudinal and multilevel analysis. Journal of Vocational Behavior, 77(1), 50-57. doi:10.1016/j.jvb.2010.02.006

Oliveira, I. M., Taveira, M. C., \& Neves, L. F. (2014). Sensibilizar professores para o desenvolvimento de carreira dos alunos: Relato de uma experiência. Psicologia: Ciência e Profissão, 34(2), 512523. doi:10.1590/1982-3703001612013

Paa, H. K., \& McWhirter, E. H. (2000). Perceived influences on high school students' current career expectations. Career Development Quarterly, 49(1), 29-44. doi:10.1002/j.2161-0045.2000.tb00749.x

Perry, J. C., Liu, X., \& Pabian, Y. (2010). School engagement as a mediator of academic performance among urban youth: The role of career preparation, parental career support, and teacher support. The Counseling Psychologist, 38(2), 269-295. doi: 10.1177/0011000009349272

Pinto, H. R., Taveira, M. C., \& Fernandes, M. E. (2003). Os professores e o desenvolvimento vocacional dos estudantes. Revista Portuguesa de Educação, 16(1), 37-58. 
Ryan, R. M., Stiller, J. D., \& Lynch, J. H. (1994). Representations of relationships to teachers, parents, and friends as predictors of academic motivation and self-esteem. Journal of Early Adolescence, 14(2), 226-249. doi:10.1177/027243169401400207

Savickas, M. (2005). The theory and practice of career construction. In S. Brown \& R. Lent (Eds.), Career development and counseling: Putting theory and research to work (pp. 42-70). Hoboken, NJ: John Wiley.

Schultheiss, D. E. P. (2003). A relational approach to career counseling: Theoretical integration and practical application. Journal of Counseling and Development, 81(3), 301-310. doi:10.1002/j.1556-6678.2003. tb00257.x

Schultheiss, D. E. P. (2007). Career development in the context of children's and adolescents' relationships. In S. B. Skorikov \& W. Patton (Eds.), Career Development in Childhood and Adolescence (pp. 169-180). Rotterdam, The Netherlands: Sense Publishers.

Schultheiss, D. E. P., Palma, T., \& Manzi, A. (2005). Career development in middle childhood: A qualitative inquiry. Career Development Quarterly, 53(3), 246-262. doi:10.1002/j.2161-0045.2005. tb00994.x

Simões, M. R. (1994). Investigações no âmbito da aferição nacional do teste das Matrizes Progressivas Coloridas de Raven (M.P.C.R.) (Tese de doutoramento, Universidade de Coimbra). Consultado em https://estudogeral.sib.uc.pt/handle/10316/946

Vondracek, F., Lerner, R., \& Schulenberg, J. W. (1986). Career development: A life-span developmental approach. Hillsdale, NJ : Erlbaum.

Wall, J., Covell, K., \& MacIntyre, P. D. (1999). Implications of social supports for adolescents' education and career aspirations. Canadian Journal of Behavioural Science, 31(2), 63-71. doi:10.1037/ h0087074 